\title{
Multifunctional Curcumin-Nanocarriers Based on Host-Guest Interactions for Alzheimer Disease Diagnostic
}

Ramdani $L^{1}$, Bourboulou $\mathbf{R}^{1}$, Belkouch $\mathbf{M}^{1,2}$, Jebors $\mathrm{S}^{3}$, Tauran $\mathrm{Y}^{3}$, Parizot $\mathrm{C}^{4}$, Suwinska $\mathrm{K}^{5}$, Coleman AW' ${ }^{3}$, Duyckaerts $\mathrm{C}^{1,2}$ and Lazar AN ${ }^{1,2 *}$

${ }^{1}$ Research Center of ICM (UPMC, INSERM UMR S 975, CNRS UMR 7225), Paris, France

${ }^{2}$ Escourolle Neuropathology Laboratory, Salpetriere Hospital, Paris, France

${ }^{3}$ Multimaterials and Interfaces Laboratory (CNRS) - UMR 5615, Univ. Lyon 1, Villeurbanne 69622, France

${ }^{4}$ Department of Immunology Cellular and Tissue, CERVI INSERM U 945, Hospital Pitié Salpêtrière, 83 Boulevard Hospital, Paris, France

${ }^{5}$ Institute of Physical Chemistry PAN, Polish Academy of Science, Warsaw, Poland

\begin{abstract}
The accumulation of amyloid peptides 40 and 42 in senile plaques is one of the hallmark lesions of Alzheimer Disease (AD). Great efforts are currently made to design molecules able to target these lesions in brain, both for diagnostic and therapeutic aims. Recent studies showed that curcumin has high affinity for the amyloid deposits. Curcumin is a fluorescent molecule with wide pharmaceutical activities, including potent anti-oxidant, anti-inflammatory, and anti-carcinogenic properties. Still, its low solubility limits its clinical and preclinical use. The use of controlled stoichiometric ratios between curcumin and host molecules like Methyl- $\beta$-cyclodextrin, para-sulphonato-calix(4)arene and para-sulphonato-calix(6)arene allowed the solubilisation of curcumin and the formation of stable nanocarriers. Within the nanocarriers, curcumin was available at their surfaces, being able to interact with the environment. They showed high affinity for the amyloid deposits, strongly labeling not only the senile plaques but also the diffuse deposits of Alzheimer Disease brains. Their biocompatibility was proved on several cell lines. Moreover, they were shown to interact with the $A \beta$ peptide, reducing its aggregation and preventing its toxicity.
\end{abstract}

Keywords: Alzheimer disease; Amyloid peptide aggregation; Curcumin; Macromolecules; Nanocarriers

\section{Introduction}

Alzheimer Disease (AD) is one of the most common forms of dementia, currently affecting more than 26 million people worldwide. The two pathological features characterizing $\mathrm{AD}$ are the intraneuronal accumulation of tau protein (neurofibrillary tangles) and the extracellular aggregation of the Amyloid beta peptide, $(A \beta)$, (senile plaques). Their detection in post-mortem tissue is still indispensable for the diagnosis [1]. The A $\beta$ peptide, the main component of senile plaques, is considered to be the driving actor in the progression of Alzheimer Disease. According to the amyloid cascade hypothesis [2,3], the accumulation of $A \beta$ peptide in the brain is the initial event in the sucession of reactions that lead to cortical dysfunction. $A \beta$ plaques are present in moderate to frequent numbers in the cortical grey matter of AD patients, years before the onset of dementia. The possibility of efficiently target $\mathrm{A} \beta$ pathology in the brain is an important strategy for developing solutions for early diagnosis. Still, the development of biocompatible probes with selective affinity for the amyloid plaques is a challenge.

Several studies showed the potential of curcumin in the treatment of $\mathrm{AD}$. Curcumin not only impedes the aggregation of the amyloid peptide but is also able to disaggregate the already formed fibrils, according to in vitro studies [4-7]. Curcumin, as well as curcumin derivatives, already proved their potential for the diagnosis of $\mathrm{AD}$, being able to bind to the amyloid deposits in vitro, in vivo or on postmortem tissue [8-13]. Moreover, curcumin is known to have numerous other protective and curative properties: anti-oxidant [14-19], antiinflammatory [20-22], and anti-cancerous [23-27], confirming its potential bio-compatibility.

Despite such encouraging reports, the study of curcuminoids is severely limited by their exceedingly low bioavailability, due to their poor solubility and instability in aqueous solutions. Thus, curcuminoids are often prepared in dimethyl sulfoxide (DMSO) or methanol, and this has raised questions about their clinical efficacy. As the need for suitable probes for targetting $A \beta$ aggregates in brain at early stages of $\mathrm{AD}$ is becoming imperative, efforts have been made to improve the solubility of these compounds [28].

Nanoparticle-based delivery has the potential of rendering hydrophobic agents, such as curcumin, dispersible in aqueous media. Liposomes and polymeric nanoparticles have been widely used as delivery systems [29-33]. An alternative to increasing the water solubility of active principles is the complexation with macromolecules. Cyclodextrins [34] and calix(n)arenes [35] are the most studied classes of macrocyclic host-compounds in supramolecular chemistry [36]. The cyclodextrins ( $\alpha-, \beta-, \gamma$-cyclodextrins) and their derivatives (2-O-methyl $\beta$-CD hydroxypropyl- $\beta-\mathrm{CD}$ and hydroxypropyl- $\gamma$-CD) were already proved to complex curcumin [37-43], improving its solubility by about 100 times. Para-sulphonato-calix(4)arene was also recently shown to be able to stabilize curcumin in aqueous solutions. The improved bio-activity of curcumin-cyclodextrin complexes was confirmed by several studies [37-40,42]. Two recent studies reported on the therapeutic effect of curcumin-cyclodextrin formulation in vivo, in AD mice models $[43,44]$. Still, up to now, the possibility to solubilize curcumin with the aid of macromolecules without impeding its ability

*Corresponding author: Adina N Lazar, Escourolle Neuropathology Laboratory, Hospital de la Salpetriere, AP-HP, 47 Bd de l'Hopital 75013 Paris, France, Tel: 331-42161891; Fax: 33-1-42161899; E-mail: adina m31@yahoo.com

Received December 24, 2014; Accepted February 10, 2015; Published February 25, 2015

Citation: Ramdani L, Bourboulou R, Belkouch M, Jebors S, Tauran Y, et al. (2015) Multifunctional Curcumin-Nanocarriers Based on Host-Guest Interactions for Alzheimer Disease Diagnostic. J Nanomed Nanotechnol 6: 270. doi:10.4172/2157 7439.1000270

Copyright: @ 2015 Ramdani L, et al. This is an open-access article distributed under the terms of the Creative Commons Attribution License, which permits unrestricted use, distribution, and reproduction in any medium, provided the original author and source are credited. 
to target the amyloid peptide and the amyloid deposits, for potential application in the early diagnosis of $\mathrm{AD}$ has not been evaluated.

Here we report a new stealth and efficient solubilisation of curcumin using the para-sulphonato-calix(4)arene (SC4), the para-sulphonatocalix(6)arene (SC6) and Methyl $\beta$-cyclodextrin. These nanoparticles were able to strongly label various amyloid aggregates in $\mathrm{AD}$ brains proving their potential as trackers of AD pathology.

\section{Experimental}

\section{Materials}

Curcumin and Methyl $\beta$-cyclodextrin $(\mathrm{M} \beta \mathrm{CD})$ were obtained from SIGMA and were used as received. The para-sulphonato-calix(4)arene (SC4) and the para-sulphonato-calix(6)arene (SC6) were synthesized according to the method of Coleman et al, by direct sulphonation of calix(n)arene [45].

\section{Curcumin solubilisation}

Curcumin was solubilized in ethanol at a concentration of $3 \mathrm{mM}$. A volume of $5 \mathrm{ml}$ of macromolecules in water (at $300 \mu \mathrm{M}$ concentration) was added, drop by drop, to $500 \mu \mathrm{l}$ of curcumin solution, under continuous stirring. Thus, a final molar ratio of 1:1 between curcumin and the macromolecules was obtained. The formation of a colloidal phase is instantaneous. Stirring was allowed for $2 \mathrm{~h}$, without a cap, to completely evaporate ethanol. The so obtained complexes: $\mathrm{M} \beta \mathrm{CD}$ curcumin (M $\beta C D$-Curc), SC4-curcumin (SC4-Curc) and SC6curcumin (SC6-Curc) were kept at $4^{\circ} \mathrm{C}$ for $12 \mathrm{~h}$, on a rotating wheel. The eventual curcumin precipitate was eliminated by centrifugation at $10000 \mathrm{rpm}$ for $15 \mathrm{~min}$. The loaded quantity of curcumin within the inclusion complexes was further estimated spectrofluorimetrically in $50 \% \mathrm{EtOH}$ and was found to be of $20 \mu \mathrm{g} / \mathrm{ml}$ for all the complexes. The colloids were further stocked at $4^{\circ} \mathrm{C}$ until use.

\section{Molecular docking studies}

The 3D molecular structures of curcumin and of the three inclusion complexes were obtained from the Cambridge database. The docking was performed with AutoDock Vina software and analyzed by PYMOL of the Scripps Research Institute. The receptor (M $\beta C D$, SC4 or SC6) and the ligand (curcumin) were built up independently and the inclusion complex model was determined by energy minimization.

\section{Characterization of the complexes}

The size of the supramolecular structures was estimated by dynamic light scattering, using a Malvern Nanosizer (particle sizer and $\zeta$-potential analyser), with a $625 \mathrm{~nm}$ laser beam. The intensity autocorrelation functions of the light scattered at a fixed angle of $173^{\circ}$ gave the size and the polydispersity indices. Transmission electron microscopy was employed to analyse the morphology of the complexes. Volumes of $1 \mu \mathrm{l}$ of each colloid were deposited on formvar-coated copper TEM grids. The morphology of samples was observed under JEOL-1210 transmission electron microscope (JEOL, Tokyo, Japan) operating at $60 \mathrm{kV}$.

The monodispersity of the supramolecular structures and the stability in time were evaluated by means of flow cytometry, using a Gallios $^{\oplus}$ cytometer (Beckman Coulter), at room temperature.

\section{Alzheimer disease cases}

AD patients enrolled in a brain donation program of the Brain Bank "GIE NeuroCEB" run by a consortium of Patients Associations (including France Alzheimer Association) were employed for this study. The consent for the research utilization was signed by the patient himself, or the next of kin, in accordance with the French Bioethical Laws. The corpse was transported to the mortuary of a University Hospital belonging to the Neuro CEB network at the time of death. The brain was removed; one hemisphere was fixed in buffered $4 \%$ formaldehyde for the neuropathological diagnosis of $\mathrm{AD}$; the other was immediately sliced. Samples from the superior temporal gyrus (Brodmann area 22) were mounted on a cork piece, dipped in isopentane cooled by liquid nitrogen and kept in a deep freezer at $-80^{\circ} \mathrm{C}$ until use. The present study has been approved by the ad hoc committee of the Brain Bank.

\section{Diagnosis}

Formalin-fixed $5 \mu \mathrm{m}$ thick sections, from multiple areas of the brain, including hippocampus and isocortical Brodmann area 22, were used for the diagnosis. The diagnosis of $\mathrm{AD}$ was confirmed by immunostaining with anti-A $\beta$ antibody (6F3D clone; Dako, Trappes France) and anti-tau antibody (polyclonal rabbit anti-tau; Dako; Trappes code number A 0024). The diagnosis criteria of the NIAReagan Institute were used [46].

\section{Affinity of curcumin complexes for the amyloid deposits}

Sections from the temporal isocortex (superior temporal gyrus) of three AD subjects (Braak neurofibrillary stage VI, Thal phase 5), containing numerous amyloid deposits and neurofibrillary tangles were used for the study. Post-mortem frozen $10 \mu \mathrm{m}$ thick sections were prepared with the aid of a Leica cryostat. The samples were hydrated for 5 min with Phosphate Buffer Saline (PBS) and then incubated $2 \mathrm{~h}$ with 200 $\mu \mathrm{l}$ of the curcumin-macromolecules solution at $1 \mu \mathrm{g} / \mathrm{ml}$. Subsequently, the samples were gently washed in PBS and mounted with a medium minimizing fading of the fluorescence (Dako Fluorescence Mounting Medium). In order to confirm the positive staining of amyloid deposits by curcumin-macromolecules complexes, $\mathrm{A} \beta$ immunohistochemistry was also performed before incubation with curcumin. After acetone fixation and PBS washing, the sections were incubated for $4 \mathrm{~h}$ in 200 $\mu \mathrm{l}$ solution of $6 \mathrm{~F} / 3 \mathrm{D}$ antibody (Dako), at $1 / 200$ dilution. The samples were washed 3 times with PBS and incubated for other $2 \mathrm{~h}$ with the secondary antibody bearing the chromogen red Cy3. Following the immunostaining, the samples were further washed in PBS before incubation with curcumin-macromolecule complexes, as previously described. The colocalization was examined using $488 \mathrm{~nm}$ (to detect the staining of curcumin) and $543 \mathrm{~nm}$ (for Cy3 detection) excitation wavelengths, the signals being collected between $540-550 \mathrm{~nm}$ and 565$580 \mathrm{~nm}$, respectively. Colocalization was indicated by a yellow color on the "merged" images.

\section{Cytotoxicity assay}

To evaluate the cytotoxicity of curcumin complexes, control SHSY5Y cells (human neuroblastoma cells), HEK cells (from Human Embryonic Kidney), hAPP ${ }_{\text {sw }}$ SH-SY5Y and hAPP ${ }_{\text {sw }}$ HEK cells - cells stably overexpressing the human APP gene (hAPP) bearing the Swedish mutation causing familial Alzheimer disease - were used. The cells were seeded in 96 multiwell culture plates and grown in DMEM-F12 media containing $10 \%$ of Fetal Calf Serum (FCS) until approximately $80 \%$ confluence. The growth medium was then discarded and the cells were incubated for $24 \mathrm{~h}$ in a culture medium enriched in curcumin complexes (final concentration of curcumin $200 \mathrm{ng} / \mathrm{ml}$ ). The cytotoxicity was assessed by means of MTT test, based on the conversion of tetrazolium salt into a purple formazan product [47]. 
Citation: Ramdani L, Bourboulou R, Belkouch M, Jebors S, Tauran Y, et al. (2015) Multifunctional Curcumin-Nanocarriers Based on Host-Guest Interaction sfor Alzheimer Disease Diagnostic. J Nanomed Nanotechnol 6: 270. doi:10.4172/2157-7439.1000270

Page 3 of 7

\section{Rescuing effect}

Control SH-SY5Y cells were used for determining the capacity of curcumin-macromolecule complexes to rescue the toxicity of $\mathrm{A} \beta$ 42. Cells were seeded in 96 multiwell culture plates and grown until approximately $80 \%$ confluence. At this point, the growth medium was replaced by a new medium to which a freshly prepared solution of $A \beta 42$ (purchased from GenicBio) in PBS (100 ng/ml) was added. The culture medium was also supplemented or not with curcumin-macromolecule complexes, with a final concentration of curcumin of $200 \mathrm{ng} / \mathrm{ml}$. The cell viability was assessed by an MTT test, the experiments being carried six times in triplicates.

\section{Aggregation assay}

A solution of $5 \mu \mathrm{M} \mathrm{A} \beta 42$ in water was freshly prepared and allowed to spontaneously aggregate at $20^{\circ} \mathrm{C}$. A volume/volume solution of curcumin-macromolecule complexes $(5 \mu \mathrm{g} / \mathrm{ml}$ final concentration in curcumin), or PBS (used as control) was added to the $A \beta 42$ solution. The effect of curcumin on the aggregation was measured by Thioflavin $T$ assay [48], monitoring the fluorescence between 410-470 nm excitation and $475-495 \mathrm{~nm}$ emission wavelengths.

\section{Results}

\section{Structural characterization of curcumin-macromolecule complexes}

The M $\beta C D$ macromolecule is a 7 membered $\alpha, 1-4$ glucose cyclic molecule, while SC4 and SC6 are macrocycles composed of respectively 4 and 6 phenolic rings, functionalized at the upper rim by sulphonate groups, favoring numerous electrostatic interactions, besides the hydrophobic aromatic interactions. The structural characteristics of curcumin and of its host macromolecules, as well as the model inclusion complexes are presented in Figure 1. In the complex with $M \beta C D$, one of the phenolic rings of the curcumin is engaged in the macrocycle, stabilized by hydrogen bonds with the hydroxyl groups. According to the molecular docking, SC4 is able to complex 2 curcumin molecules. One of the molecules, cinctured the macrocycle, two phenolic rings being involved in $\pi-\pi$ interactions with the host. Another curcumin

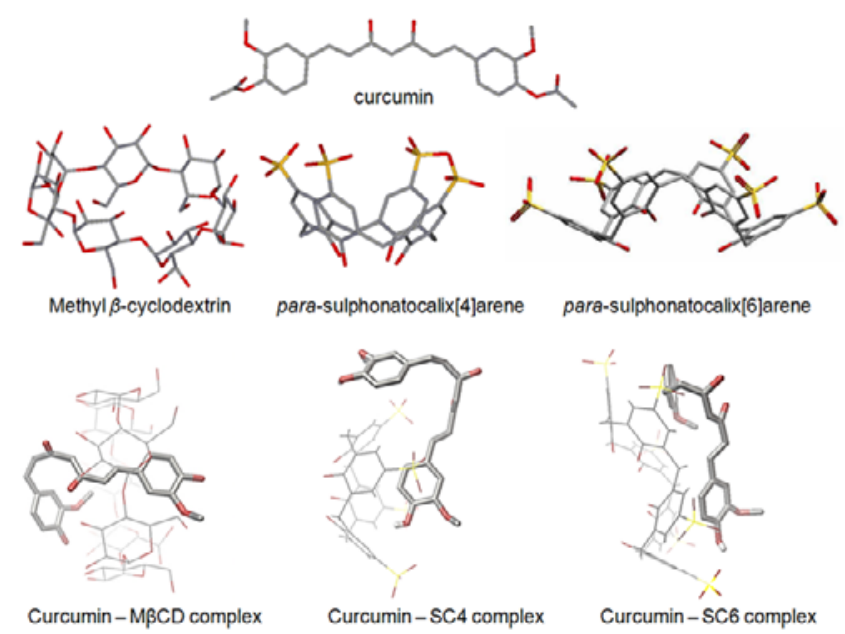

Figure 1: Molecular structure of curcumin, of the three macromolecules used for curcumin solubilization and of the inclusion complexes formed by curcumin with the host-molecules; grey color represents carbon atoms, red color represents the oxygen atoms and the yellow color represents the sulfur atoms.

\begin{tabular}{|l|c|c|}
\hline Complex & Size average $\mathbf{( n m )}$ & Polydispersity Index \\
\hline Curcumin-M $\beta C D$ & 45 & 0,33 \\
\hline Curcumin-SC4 & 68 & 0,42 \\
\hline Curcumin-SC6 & 75 & 0,31 \\
\hline
\end{tabular}

Table 1: Characterization of curcumin-macromolecule complexes by dynamic light scattering; average of three runs, rounded to the nearest number.

a)

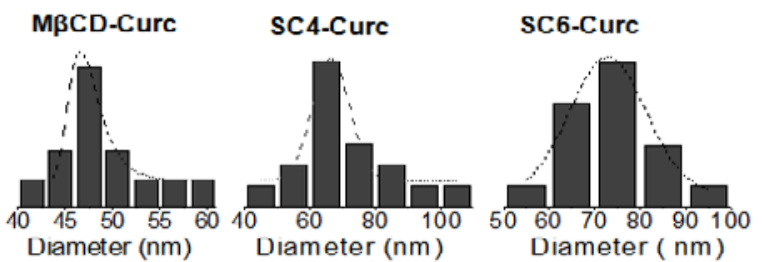

b)
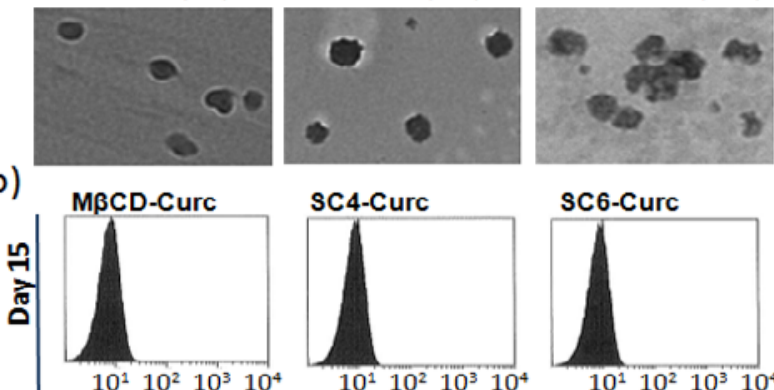

SC4-Curc

sc6-Curc
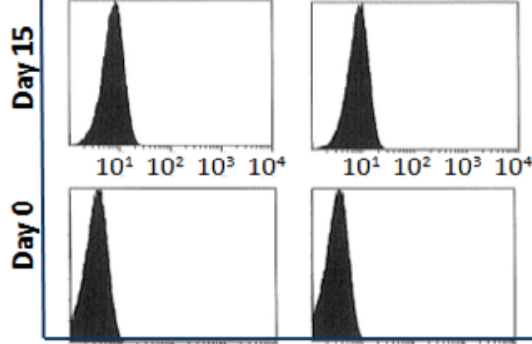

$\begin{array}{llll}10^{1} & 10^{2} & 10^{3} & 10^{4}\end{array}$

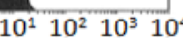

$10^{1} 10^{2} 10^{3} 10^{4}$

$10^{1} 10^{2} 10^{3} 10^{4}$

$10^{1} 10^{2} 10^{3} 10^{4}$

Figure 2: Characterization of curcumin-macromolecule complexes: a) Morphological analysis by TEM revealed a monodisperse population of nanoparticles with several tens of nanometers in size; b) stability in time of curcumin-macromolecule complexes, as revealed by flow cytometry; A. U. = Arbitrary Units.

was partly encapsulated by $\pi-\pi$ stacking with one of the phenol groups, and by hydrogen bonds with the suphonate groups of the host; the other moiety of the molecule is free of constraints. In a similar way SC6 formed a complex with 2 curcumin molecules, one at the upper rim of the calix(6)arene, the other one at the lower rim. Both guest molecules are stabilized by $\mathrm{H}$-bonds interactions with the hydroxyl and sulphonate groups of the host and by $\pi-\pi$ stacking interactions of two phenolic rings with the calix(6)arene macrocycle. Given the flexibility of the curcumin, but also of the macromolecules, other structures for the complexes are possible.

\section{Characterization of curcumin-macromolecule complexes}

The size of the curcumin-macromolecule complexes was estimated by dynamic light scattering and was found to be of $45 \mathrm{~nm}$ for $\mathrm{M} \beta \mathrm{CD}$ curcumin and around $70 \mathrm{~nm}$ for SC4/SC6-curcumin (Table 1). The morphology of the curcumin-macromolecule complexes was determined by transmission electron microscopy. Monodispersed nanoparticles of a diameter between 45 and $75 \mathrm{~nm}$ were characteristic for the three complexes (Figure 2a). Adapted flow cytometry analysis at different time intervals proved that the size of the nanoparticles diminished slightly in time (several $\mathrm{nm}$ ), but they remain monodisperse for more than 15 days (Figure $2 b$ ). 
Citation: Ramdani L, Bourboulou R, Belkouch M, Jebors S, Tauran Y, et al. (2015) Multifunctional Curcumin-Nanocarriers Based on Host-Guest Interaction sfor Alzheimer Disease Diagnostic. J Nanomed Nanotechnol 6: 270. doi:10.4172/2157-7439.1000270

\section{Post-mortem AD brain tissue}

The affinity of curcumin nanocarriers for the amyloid deposits was tested on post-mortem brain samples of three $\mathrm{AD}$ patients. The amyloid pathology of the cases chosen for the study was confirmed by immunohistochemistry with an antibody frequently used for the detection of the $\mathrm{A} \beta$ deposits, the $6 \mathrm{~F} 3 \mathrm{D}$ antibody. The cases presented numerous amyloid deposits, from diffuse deposits (an early stage of the senile plaque) to mature senile plaques (Duyckaerts 1990) (Figure 3).

\section{Affinity of curcumin-nanocarriers for amyloid deposits in} human AD brains

Numerous amyloid deposits were specifically labeled by the three curcumin nanocarriers. The specificity was confirmed by the double labeling with $6 \mathrm{~F} 3 \mathrm{D}$ antibody (directed against the $\mathrm{A} \beta$ deposits). The senile plaques were strongly labeled by the curcumin nanocarriers (Figure 4). The nanocarriers also labeled $65 \%$ of the diffuse deposits.
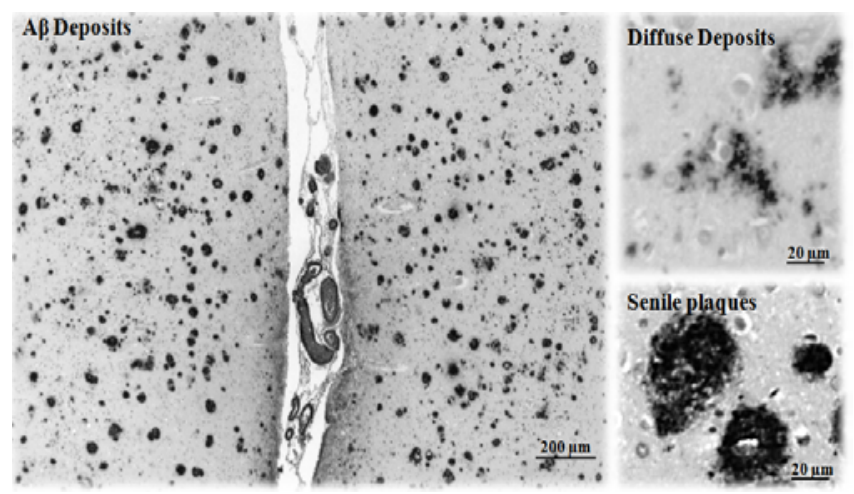

Figure 3: Micrographs of a post-mortem superior temporal cortex sample of an AD patient revealed by $6 F 3 D$ anti-A $\beta$ antibody: numerous senile plaques and diffuse deposits are present in the cortical area.
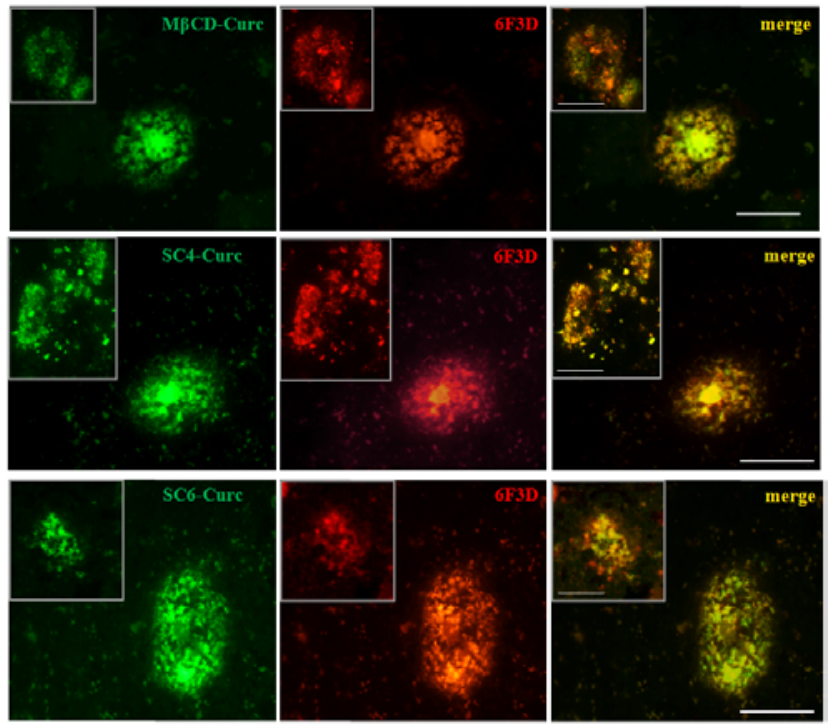

Figure 4: Affinity of curcumin-nanocarriers for amyloid deposits; curcumin labeling in green, 6F3D labeling (specific for $A \beta$ deposits) in red and the colocalization of the labeling, on the merged image, in yellow; the insert shows the labeling of the diffuse deposits); scale bar $=50 \mu \mathrm{m}$.
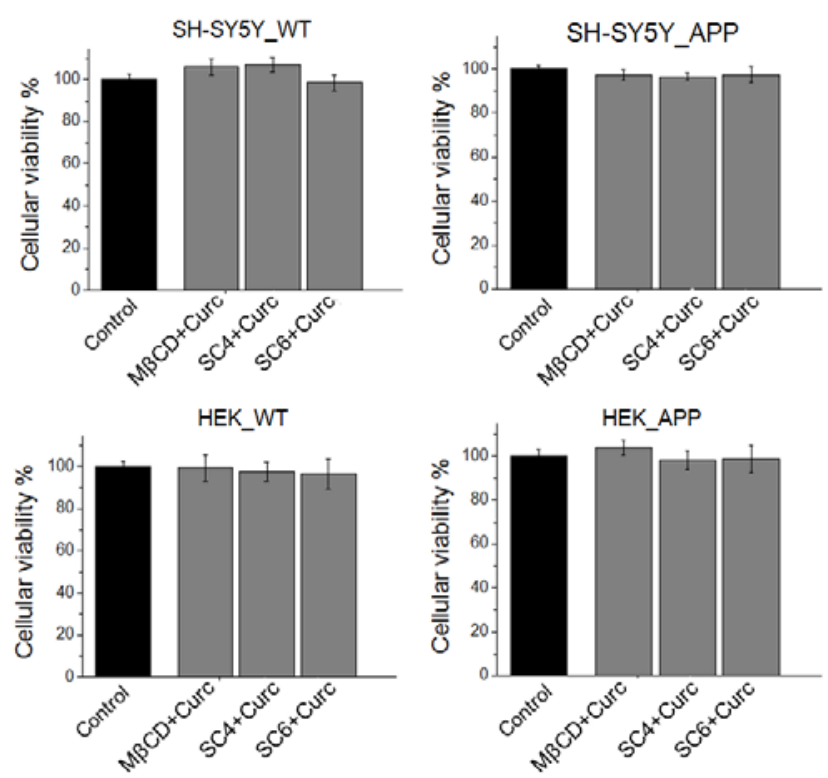

Figure 5: Effect of curcumin-nanocarriers on the cellular viability of several cell lines after $24 \mathrm{~h}$ of incubation: wild type lines of SH-SY5Y human neuroblastoma and KEK embryonic kidney and their APP mutated lines (stably expressing swap mutation).

The macromolecules alone were not fluorescent and showed no labeling of the deposits (results not shown).

\section{Biocompatibility of curcumin-complexes as tracers for the amyloid deposits}

The cytotoxicity of M $3 \mathrm{CD}$, SC4 and of SC6 and of the curcuminnanocarriers formed with these three macromolecules was evaluated by MTT test on human neuroblastoma SH-SY5Y and embryonic kidney HEK cell, wild type and APP (stably overexpressing hAPP bearing the Swedish mutation). Neither the macromolecules alone (results not shown) nor the three curcumin-nanocarriers showed a toxic effect (Figure 5).

\section{Effect on $A \beta$ toxicity and aggregation}

The toxic effect of $A \beta 42$ peptide in the presence of curcuminnanocarriers was evaluated on SH-SY5Y wild type neurblastoma cells, over $24 \mathrm{~h}$ of incubation. The three nanocarriers showed a significant rescuing effect: the viability of cells submitted to $A \beta 42$ was increased from $76 \%$ to $93 \%$ by M 3 CD-Curc, $97 \%$ by SC4-Curc and $96 \%$ by SC6Curc (Figure 6a). The link between the rescuing effect of curcuminnanocarriers and the ability of curcumin to impede peptide aggregation was evaluated with a thioflavin $\mathrm{T}$ assay. The three curcuminnanocarriers showed a strong inhibitory effect on peptide fibrillization. A relative estimation of the inhibition efficiency showed a reduction of the fibrillar content to: $62 \%$ by M $\beta C D$-Curc, $39 \%$ by SC4-Curc and $56 \%$ by SC6-Curc (Figure $6 \mathrm{~b}$ ).

\section{Discussion}

A new delivery system, based on the use of macromolecules for solubilizing curcumin, is presented in this study. Hydrosoluble calix(4) arenes, SC4 and SC6, bearing 4 or 6 aromatic cycles functionalized at the upper rim with sulphonate groups, and $\mathrm{M} \beta \mathrm{CD}$ efficiently solubilized curcumin. The curcumin-macromulecule complexes were organized 
a)

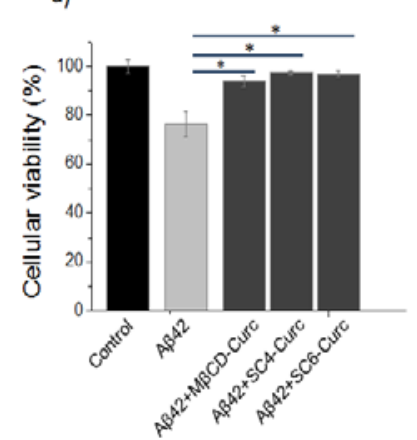

b)

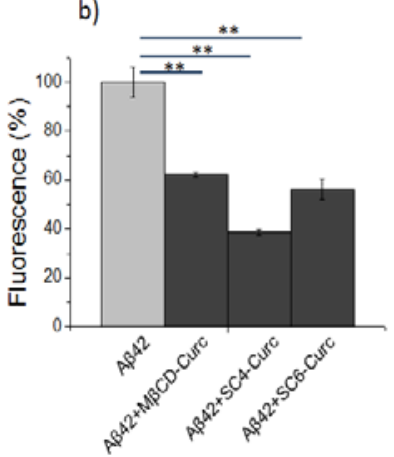

Figure 6: Effect of curcumin-nanocarriers on $A \beta 42$ toxicity and aggregation: a) Rescuing effect of curcumin-nanocarriers on SH-SY5Y human neuroblastoma treated with $A \beta 42 ; b)$ Effect of curcumin complexes on the fibrillization of $A \beta$ peptide; ( $p$-values $<0,05$ for the bilateral $t$ test).

into nanoparticles of tens of nanometers in diameter, stable in time, for at least 15 days. Within the generated nanocarriers, the ability of curcumin to interact with the amyloid deposits in post-mortem brain samples of $\mathrm{AD}$ patients was conserved. Both mature senile plaques and diffuse deposits-the kinetic precursor of the senile plaques-were labeled by the three curcumin-nanocarriers. The strong affinity for the mature $A \beta$ deposits and especially for the early aggregates points them as potential radiotracers or MRI markers for early diagnosis in $\mathrm{AD}$. In view of this, several in vitro tests proved the biocompatibility of these curcumin nanocarriers: they were shown to be non-toxic on different cells lines, to significantly rescue the toxicity induced by $\mathrm{A} \beta$ 42 aggregates and to limit the fibrillization of $\mathrm{A} \beta$ peptide.

Alzheimers disease is a frequent form of dementia, $A \beta$ plaques and NFTs playing a major role in the development of this disease. Significant evidences suggest that progressive accumulation of $A \beta$ in limbic and association cortices precedes the neurodegeneration of tau and the cascade of biochemical and cellular modifications in the brain. The development of strategies to detect $A \beta$ pathological changes in vivo, in the early stages of $\mathrm{AD}$ is essential. Currently, no imaging techniques capable of early detection of $\mathrm{AD}$ are available in clinics. The PET tracer [11], Pittsburg Compound (PIB) is employed for clinical diagnosis since 2004, after the first human study [49]. Other ${ }^{18} \mathrm{~F}$-amyloid tracers have been developed since, but their specificity is questioned. Moreover, these tracers have low affinity for the diffuse deposits, the diagnosis indicated being negative in the absence of senile plaques. Magnetic Resonance Imaging (MRI) is also a powerful tool for clinical and biological imaging able to map structure and function with excellent spatial resolution. Numerous amyloid scintigraphic and magnetic probes have been or are being developed. Still, few are fulfilling all the requirements in order to be employed for clinical diagnosis: bioavailable, non-toxic and target specific.

Numerous studies confirmed the potential of curcumin in the treatment of $\mathrm{AD}[7,44,50-54]$ and lately, in early diagnosis [5,10,5558]. The lack of complete success of curcumin essays resides in its low bioavailability, related to its insolubility in aqueous solutions. Hugh efforts were made to improve its solubility, from chemistry to nanotechnology, either by functionalizing the basic molecule with ionic functions or by conjugation of small molecules (particularly amino acids [59]) or by designing nanoparticles based systems, like liposomes [30,60-62], solid lipid nanoparticles [31,33,63-65], or polymeric nanoparticles $[32,44,62,66-70]$. These types of nano-formulations are

generally based on the inner encapsulation of curcumin. Formulations designed to solubilize curcumin by conserving its ability to bind $A \beta$ peptide or to target the amyloid deposits are few [30,60] and they concern liposomes incorporating phopsphatidyl serine covalently linked with curcumin through the polar head.

Solubilization of curcumin by means of inclusion complexes represents a convenient alternative for increasing curcumin bioavailability concomitantly with preserving it available for targeting and interaction with the environment. Among the various existing drug carriers $[35,71,72]$, cyclodextrins have been almost exclusively used for the solubilization of curcumin [37,41-43]. The stoichiometric ratio between cyclodextrins and curcumin was reported to be $2: 1[37,73]$, suggesting that each of the two phenolic rings of curcumin would be sheltered by the lipophilic cavity of two cyclodextrins. By controlling the molar concentration of each component, a complex ratio of 1:1 cyclodextrin/curcumin can be obtained $[38,41]$. Such curcumincyclodextrin complexes behave like a lipophilic supramolecule, being able to self-assemble into vesicular nanostructures in which curcumin is believed to be confined at the interior vesicle [40]. The potential of cyclodextrin-solubilized curcuminoids in the treatment of $\mathrm{AD}$ was already evaluated by the group of Quitschke et al.: by using 2-hydroxypropyl-gcyclodextrin, they showed that cyclodextrinsolubilized curcuminoids are able to lower the amyloid plaque load in $\mathrm{AD}$ transgenic mice [43]. The study focused mainly on the metabolism of curcumin and curcuminoids. But could such formulations preserve the targeting ability of curcumin? This question was not examined before, to our knowledge.

In this study, we have proved that $\mathrm{M} \beta \mathrm{CD}$-curcumin complex could self-assemble into nanoparticles in a way that retains, at least partially, the curcumin at the surface of the nanoparticles. Likewise curcuminpara-sulphonato-calix(4)arene and curcumin-para-sulphonatocalix(6)arene complexes self-assembled into nanoparticular systems, with curcumin at the surface of the particles. Several approaches have confirmed the availability of curcumin at the surface of these nanoparticles: the nanoparticles prevented $A \beta 42$ induced toxicity; they were able to interact with the $\mathrm{A} \beta$ peptide, reducing its aggregation and they showed high affinity for the amyloid deposits, strongly labeling not only the senile plaques but also the diffuse deposits of $A \beta$ on brain tissue of post-mortem $\mathrm{AD}$ patients.

The potential of such curcumin-nanocarriers in early diagnosis as well as in the treatment of AD is to be considered as few reports deal with both therapeutic and preventive approach. The use of not only non-toxic, but also pro-active amyloid tracers may offer the possibility of an early diagnosis combined with a subsequent treatment effect, by preventing the toxicity due to $A \beta$ aggregates, limiting the progression of the aggregation and thus, the evolution of the disease.

\section{Acknowledgement}

This work was supported by the European Community's Seventh Framework Programme (FP7/2007-2013) under grant agreement no. 212043. ANL was partially funded by Ministry of Health PHRC 2010 (France). This work has received financial support from the program "Investissements d'Avenir" ANR-10-IAIHU-06 The expertise of the technical staff of Escourolle Neuropathology Laboratory as well as the collaboration of the "Cell Imaging Platform" at Brain and Spine Institute are greatly acknowledged. The brain tissues were kindly provided by the GIE NeuroCEB brain bank (France Alzheimer, France Parkinson, ARSEP, CSC).

\section{References}

1. Hyman BT, Phelps CH, Beach TG, Bigio EH, Cairns NJ, et al. (2012) National Institute on Aging-Alzheimer's Association guidelines for the neuropathologic assessment of Alzheimer's disease. Alzheimers Dement 8: 1-13. 
Citation: Ramdani L, Bourboulou R, Belkouch M, Jebors S, Tauran Y, et al. (2015) Multifunctional Curcumin-Nanocarriers Based on Host-Guest Interaction sfor Alzheimer Disease Diagnostic. J Nanomed Nanotechnol 6: 270. doi:10.4172/2157-7439.1000270

2. Hardy J (1999) The shorter amyloid cascade hypothesis. Neurobiol Aging 20: 85.

3. Hardy J (2002) Testing times for the "amyloid cascade hypothesis". Neurobiol Aging 23: 1073-1074

4. Ono K, Hasegawa K, Naiki H, Yamada M (2004) Curcumin has potent antiamyloidogenic effects for Alzheimer's beta-amyloid fibrils in vitro. J Neurosci Res 75: 742-750.

5. Yang F, Lim GP, Begum AN, Ubeda OJ, Simmons MR, et al. (2005) Curcumin inhibits formation of amyloid beta oligomers and fibrils, binds plaques, and reduces amyloid in vivo. J Biol Chem 280: 5892-5901.

6. Zhang C, Browne A, Child D, Tanzi RE (2010) Curcumin decreases amyloidbeta peptide levels by attenuating the maturation of amyloid-beta precursor protein. J Biol Chem 285: 28472-28480.

7. Begum AN, Jones MR, Lim GP, Morihara T, Kim P, et al. (2008) Curcumin structure-function, bioavailability, and efficacy in models of neuroinflammation and Alzheimer's disease. J Pharmacol Exp Ther 326: 196-208.

8. Lee I, Yang J, Lee JH, Choe YS (2011) Synthesis and evaluation of 1-(4-[Ầ â, F] fluoroethyl)-7-(4'-methyl)curcumin with improved brain permeability for $\beta$-amyloid plaque imaging. Bioorg Med Chem Lett 21: 5765-5769.

9. Yanagisawa D, Amatsubo T, Morikawa S, Taguchi H, Urushitani M, et al. (2011) In vivo detection of amyloid beta deposition using (1)F magnetic resonance imaging with a (1)F-containing curcumin derivative in a mouse model of Alzheimer's disease. Neuroscience 184: 120-127.

10. Koronyo-Hamaoui M, Koronyo $\mathrm{Y}$, Ljubimov AV, Miller CA, Ko MK, et al. (2011) Identification of amyloid plaques in retinas from Alzheimer's patients and noninvasive in vivo optical imaging of retinal plaques in a mouse model. Neuroimage 54 Suppl 1: S204-217.

11. Ray B, Lahiri DK (2009) Neuroinflammation in Alzheimer's disease: differen molecular targets and potential therapeutic agents including curcumin. Curr Opin Pharmacol 9: 434-444.

12. Cheng AL, Hsu CH, Lin JK, Hsu MM, Ho YF, et al. (2001) Phase I clinical trial of curcumin, a chemopreventive agent, in patients with high-risk or pre-malignant lesions. Anticancer Res 21: 2895-2900.

13. Holt PR, Katz S, Kirshoff R (2005) Curcumin therapy in inflammatory bowe disease: a pilot study. Dig Dis Sci 50: 2191-2193.

14. Muthenna P, Suryanarayana P, Gunda SK, Petrash JM, Reddy GB (2009) Inhibition of aldose reductase by dietary antioxidant curcumin: mechanism of inhibition, specificity and significance. FEBS Lett 583: 3637-3642.

15. Lin J, Zheng S, Chen A (2009) Curcumin attenuates the effects of insulin on stimulating hepatic stellate cell activation by interrupting insulin signaling and attenuating oxidative stress. Lab Invest 89: 1397-1409.

16. BelviranlÄ M, Okudan N, AtalÄk KE, Ãz M (2013) Curcumin improves spatial memory and decreases oxidative damage in aged female rats. Biogerontology 14: $187-196$

17. Aldebasi YH, Aly SM, Rahmani AH (2013) Therapeutic implications of curcumin in the prevention of diabetic retinopathy via modulation of anti-oxidant activity and genetic pathways. Int J Physiol Pathophysiol Pharmacol 5: 194-202.

18. Kawanishi N, Kato K, Takahashi M, Mizokami T, Otsuka Y, et al. (2013) Curcumin attenuates oxidative stress following downhill running-induced muscle damage. Biochem Biophys Res Commun 441: 573-578.

19. Meng B, Li J, Cao H (2013) Antioxidant and antiinflammatory activities of curcumin on diabetes mellitus and its complications. Curr Pharm Des 19: 2101 2113.

20. Panahi Y, Sahebkar A, Parvin S, Saadat A (2012) A randomized controlled tria on the anti-inflammatory effects of curcumin in patients with chronic sulphur mustard-induced cutaneous complications. Ann Clin Biochem 49: 580-588

21. Colitti M, Gaspardo B, Della Pria A, Scaini C, Stefanon B (2012) Transcriptome modification of white blood cells after dietary administration of curcumin and non-steroidal anti-inflammatory drug in osteoarthritic affected dogs. Vet Immunol Immunopathol 147: 136-146.

22. Shehzad A, Rehman G, Lee YS (2013) Curcumin in inflammatory diseases. Biofactors 39: 69-77.

23. Rao CV, Rivenson A, Simi B, Reddy BS (1995) Chemoprevention of colon carcinogenesis by dietary curcumin, a naturally occurring plant phenolic compound. Cancer Res 55: 259-266.
24. Huang CZ, Huang WZ, Zhang G, Tang DL (2013) In vivo study on the effects of curcumin on the expression profiles of anti-tumour genes (VEGF, CyclinD1 and CDK4) in liver of rats injected with DEN. Mol Biol Rep 40: 5825-5831.

25. Shehzad A, Park JW, Lee J, Lee YS (2013) Curcumin induces radiosensitivity of in vitro and in vivo cancer models by modulating pre-mRNA processing factor 4 (Prp4). Chem Biol Interact 206: 394-402.

26. Yu Y, Zhang X, Qu L (2014) The anti-tumor efficacy of curcumin when delivered by size/charge-changing multistage polymeric micelles based on amphiphilic poly( $\hat{I}^{2}$-amino ester) derivates. Biomaterials 35: 3467-3479.

27. Saxena V, Hussain MD (2013) Polymeric mixed micelles for delivery of curcumin to multidrug resistant ovarian cancer. J Biomed Nanotechnol 9: 11461154

28. Quitschke WW (2008) Differential solubility of curcuminoids in serum and albumin solutions: implications for analytical and therapeutic applications. BMC Biotechnol 8: 84

29. Basnet $P$, Hussain $H$, Tho I, Skalko-Basnet N (2012) Liposomal delivery system enhances anti-inflammatory properties of curcumin. J Pharm Sci 101: 598-609.

30. Mourtas S, Canovi M, Zona C, Aurilia D, Niarakis A, et al. (2011) Curcumindecorated nanoliposomes with very high affinity for amyloid-îl21-42 peptide. Biomaterials 32: 1635-1645.

31. Kakkar V, Mishra AK, Chuttani K, Kaur IP (2013) Proof of concept studies to confirm the delivery of curcumin loaded solid lipid nanoparticles (C-SLNs) to brain. Int J Pharm 448: 354-359.

32. Le Droumaguet B, Nicolas J, Brambilla D, Mura S, Maksimenko A, et al. (2012) Versatile and efficient targeting using a single nanoparticulate platform: application to cancer and Alzheimer's disease. ACS Nano 6: 5866-5879.

33. Vandita K, Shashi B, Santosh KG, Pal KI (2012) Enhanced apoptotic effect of curcumin loaded solid lipid nanoparticles. Mol Pharm 9: 3411-3421.

34. Szejtli J1 (1998) Introduction and General Overview of Cyclodextrin Chemistry. Chem Rev 98: 1743-1754.

35. Perret F, Lazar AN, Coleman AW (2006) Biochemistry of the para-sulfonatocalix[n]arenes. Chem Commun (Camb) : 2425-2438.

36. Steed JW, Atwood JL (2009) Supramolecular Chemistry

37. Dandawate PR, Vyas A, Ahmad A, Banerjee S, Deshpande J, et al. (2012) Inclusion complex of novel curcumin analogue CDF and $\hat{i}^{2}$-cyclodextrin (1:2) and its enhanced in vivo anticancer activity against pancreatic cancer. Pharm Res 29: 1775-1786.

38. Tan Q, Li Y, Wu J, Mei H, Zhao C, et al. (2012) An optimized molecular inclusion complex of diferuloylmethane: enhanced physical properties and biological activity. Int J Nanomedicine 5: 5385-5393.

39. Tomren MA, Másson M, Loftsson T, Tønnesen HH (2007) Studies on curcumin and curcuminoids XXXI. Symmetric and asymmetric curcuminoids: stability, activity and complexation with cyclodextrin. Int J Pharm 338: 27-34.

40. Yallapu MM, Jaggi M, Chauhan SC (2010) beta-Cyclodextrin-curcumin selfassembly enhances curcumin delivery in prostate cancer cells. Colloids Surf B Biointerfaces 79: 113-125.

41. Baglole KN ,Boland PG, Wagne BD (2005) PFluorescence enhancement of curcumin upon inclusion into parent and modified cyclodextrins. Journal of Photochemistry and Photobiology A: Chemistry 173: 230-237.

42. Hegge AB, Vukicevic M, Bruzell E, Kristensen S, Tønnesen HH (2013) Solid dispersions for preparation of phototoxic supersaturated solutions for antimicrobial photodynamic therapy (aPDT): Studies on curcumin and curcuminoides L. Eur J Pharm Biopharm 83: 95-105.

43. Quitschke WW, Steinhauff N, Rooney J (2013) The effect of cyclodextrinsolubilized curcuminoids on amyloid plaques in Alzheimer transgenic mice: brain uptake and metabolism after intravenous and subcutaneous injection. Alzheimers Res Ther 5: 16

44. Cheng KK, Yeung CF, Ho SW, Chow SF, Chow AH, et al. (2013) Highly stabilized curcumin nanoparticles tested in an in vitro blood-brain barrier mode and in Alzheimer's disease Tg2576 mice. AAPS J 15: 324-336.

45. Shinkai S, Mori S, Koreishi H, Tsubaki T, Manabe O (1986) Hexasulfonated calix[6]arene derivatives: a new class of catalysts, surfactants, and host molecules. J Am Chem Soc 108: 2409-2416. 
Citation: Ramdani L, Bourboulou R, Belkouch M, Jebors S, Tauran Y, et al. (2015) Multifunctional Curcumin-Nanocarriers Based on Host-Guest Interaction sfor Alzheimer Disease Diagnostic. J Nanomed Nanotechnol 6: 270. doi:10.4172/2157-7439.1000270

46. (1997) Consensus recommendations for the postmortem diagnosis of Alzheimer's disease. The National Institute on Aging, and Reagan Institute Working Group on Diagnostic Criteria for the Neuropathological Assessment of Alzheimer's Disease. Neurobiol Aging 18: S1-2.

47. Mosmann T (1983) Rapid colorimetric assay for cellular growth and survival: application to proliferation and cytotoxicity assays. J Immunol Methods 65: 55-63.

48. LeVine H 3rd1 (1993) Thioflavine T interaction with synthetic Alzheimer's disease beta-amyloid peptides: detection of amyloid aggregation in solution. Protein Sci 2: 404-410.

49. Klunk WE, Engler H, Nordberg A, Wang Y, Blomqvist G, et al. (2004) Imaging brain amyloid in Alzheimer's disease with Pittsburgh Compound-B. Ann Neurol 55: 306-319.

50. Dkhar P, Sharma R (2010) Effect of dimethylsulphoxide and curcumin on protein carbonyls and reactive oxygen species of cerebral hemispheres of mice as a function of age. Int J Dev Neurosci 28: 351-357.

51. Frautschy SA, Hu W, Kim P, Miller SA, Chu T, et al. (2001) Phenolic antiinflammatory antioxidant reversal of Abeta-induced cognitive deficits and neuropathology. Neurobiol Aging 22: 993-1005.

52. Villaflores OB, Chen YJ, Chen CP, Yeh JM, Wu TY (2012) Curcuminoids and resveratrol as anti-Alzheimer agents. Taiwan J Obstet Gynecol 51: 515-525.

53. Lim GP, Chu T, Yang F, Beech W, Frautschy SA, et al. (2001) The curry spice curcumin reduces oxidative damage and amyloid pathology in an Alzheimer transgenic mouse. J Neurosci 21: 8370-8377.

54. Wang Y, Yin H, Li J, Zhang Y, Han B, et al. (2013) Amelioration of $\hat{I}^{2}$-amyloidinduced cognitive dysfunction and hippocampal axon degeneration by curcumin is associated with suppression of CRMP-2 hyperphosphorylation. Neurosci Lett 557 Pt B: 112-117.

55. Lee I, Yang J, Lee JH, Choe YS (2011) Synthesis and evaluation of 1-(4-[ÂÂa, F] fluoroethyl)-7-(4'-methyl)curcumin with improved brain permeability for $\beta$-amyloid plaque imaging. Bioorg Med Chem Lett 21: 5765-5769.

56. Ryu EK, Choe YS, Lee KH, Choi Y, Kim BT (2006) Curcumin and dehydrozingerone derivatives: synthesis, radiolabeling, and evaluation for beta-amyloid plaque imaging. J Med Chem 49: 6111-6119.

57. Sagnou M, Benaki D, Triantis C, Tsotakos T, Psycharis V, et al. (2011) Curcumin as the $\mathrm{OO}$ bidentate ligand in " $2+1$ " complexes with the [M(CO)3]+ $(\mathrm{M}=\mathrm{Re}, 99 \mathrm{mTc})$ tricarbonyl core for radiodiagnostic applications. Inorg Chem 50: $1295-1303$.

58. Narasingappa RB, Javagal MR, Pullabhatla S, Htoo HH, Rao JK, et al. (2012) Activation of Ît-secretase by curcumin-aminoacid conjugates. Biochem Biophys Res Commun 424: 691-696.

59. Lazar AN, Mourtas S, Youssef I, Parizot C, Dauphin A, et al. (2013) Curcuminconjugated nanoliposomes with high affinity for Abeta deposits: possible applications to Alzheimer disease. Nanomedicine 9: 712-721.

60. Re F, Cambianica I, Zona C, Sesana S, Gregori M, et al. (2011) Functionalization of liposomes with ApoE-derived peptides at different density affects cellular uptake and drug transport across a blood-brain barrier model. Nanomedicine 7: $551-559$.

61. Chiu SS, Lui E, Majeed M, Vishwanatha JK, Ranjan AP, et al. (2011) Differential distribution of intravenous curcumin formulations in the rat brain. Anticancer Res 31: 907-911.

62. Puglia C, Offerta A, Rizza L, Zingale G, Bonina F, et al. (2013) Optimization of curcumin loaded lipid nanoparticles formulated using high shear homogenization (HSH) and ultrasonication (US) methods. J Nanosci Nanotechnol 13: 68886893

63. Guri A, Gülseren I, Corredig M (2013) Utilization of solid lipid nanoparticles for enhanced delivery of curcumin in cocultures of HT29-MTX and Caco-2 cells. Food Funct 4: 1410-1419.

64. Wang W, Zhu R, Xie Q, Li A, Xiao Y, et al. (2012) Enhanced bioavailability and efficiency of curcumin for the treatment of asthma by its formulation in solid lipid nanoparticles. Int J Nanomedicine 7: 3667-3677.

65. Feng R, Song Z, Zhai G (2012) Preparation and in vivo pharmacokinetics of curcumin-loaded PCL-PEG-PCL triblock copolymeric nanoparticles. Int J Nanomedicine 7: 4089-4098

66. Mathew A, Fukuda T, Nagaoka Y, Hasumura T, Morimoto H, et al. (2012) Curcumin loaded-PLGA nanoparticles conjugated with Tet-1 peptide for potential use in Alzheimer's disease. PLoS One 7: e32616.

67. Doggui S, Sahni JK, Arseneault M, Dao L, Ramassamy C (2012) Neurona uptake and neuroprotective effect of curcumin-loaded PLGA nanoparticles on the human SK-N-SH cell line. J Alzheimers Dis 30: 377-392.

68. Ray B, Bisht S, Maitra A, Maitra A, Lahiri DK (2011) Neuroprotective and neurorescue effects of a novel polymeric nanoparticle formulation of curcumin (NanoCurcâ,, $\phi$ ) in the neuronal cell culture and animal model: implications for Alzheimer's disease. J Alzheimers Dis 23: 61-77.

69. Mulik RS, Mönkkönen J, Juvonen RO, Mahadik KR, Paradkar AR (2010) ApoE3 mediated poly(butyl) cyanoacrylate nanoparticles containing curcumin: study of enhanced activity of curcumin against beta amyloid induced cytotoxicity using in vitro cell culture model. Mol Pharm 7: 815-825.

70. Al-Jamal KT, Ramaswamy C, Florence AT (2005) Supramolecular structures from dendrons and dendrimers. Adv Drug Deliv Rev 57: 2238-2270.

71. Abderrezak A, Bourassa P, Mandeville JS, Sedaghat-Herati R, Tajmir-Riah HA (2012) Dendrimers bind antioxidant polyphenols and cisplatin drug. PLoS One 7: e33102.

72. Tang B, Ma L, Wang HY, Zhang GY (2002) Study on the supramolecula interaction of curcumin and beta-cyclodextrin by spectrophotometry and its analytical application. J Agric Food Chem 50: 1355-1361.

73. Tønnesen HH, Másson M, Loftsson T (2002) Studies of curcumin and curcuminoids. XXVII. Cyclodextrin complexation: solubility, chemical and photochemical stability. Int J Pharm 244: 127-135. 